DOI: $10.3895 / \mathrm{S} 2175-08582009000100003$

\section{REVISTA BRASILEIRA DE QUALIDADE DE VIDA}

\title{
Cálculo dos escores e estatística descritiva do WHOQOL-100 utilizando o Microsoft Excel
}

\section{Calculation of scores and descriptive statistics of WHOQOL-100 using Microsoft Excel}

\author{
Bruno Pedroso \\ Universidade Tecnológica Federal do Paraná - UTFPR - Ponta Grossa - Brasil, bolsista da CAPES \\ brunops3@brturbo.com.br \\ Luiz Alberto Pilatti \\ Universidade Tecnológica Federal do Paraná - UTFPR - Ponta Grossa - Brasil \\ lapilatti@utfpr.edu.br \\ Dálcio Roberto dos Reis \\ Universidade Tecnológica Federal do Paraná - UTFPR - Ponta Grossa - Brasil \\ dalcio@utfpr.edu.br
}

\begin{abstract}
Resumo
O objetivo do presente trabalho é a construção de uma ferramenta para calcular os escores e a estatística descritiva do WHOQOL-100. Para o cálculo dos resultados do WHOQOL-100, o Grupo WHOQOL recomenda a utilização do SPSS. A ferramenta proposta nesse estudo foi desenvolvida a partir do software Microsoft Excel, seguindo a sintaxe proposta pelo Grupo WHOQOL. A ferramenta foi testada por pesquisadores do Mestrado em Engenharia de Produção e do Curso Superior de Tecnologia em Análise e Desenvolvimento de Sistemas da UTFPR. Foi também submetida a uma simulação com o software SPSS, onde os resultados obtidos foram exatamente os mesmos. A sintaxe utilizada na construção da ferramenta é disponibilizada, possibilitando sua adaptação para aplicação em outros instrumentos. Conclui-se que, a partir do presente trabalho, foi possível a disponibilização de uma ferramenta gratuita, desenvolvida em uma plataforma amplamente difundida e que permite realizar aplicações do WHOQOL-100 sem necessitar a utilização do software SPSS.
\end{abstract}

Palavras-chave: Qualidade de Vida, Avaliação em Saúde, Organização Mundial da Saúde, WHOQOL-100.

\begin{abstract}
The objective of this paper is to construct a tool for calculation of the scores and descriptive statistics of WHOQOL-100. For the results calculation of WHOQOL-100, the WHOQOL Group suggests SPSS's utilization. The tool proposed in this study was developed on Microsoft Excel, following the proposed syntax by the WHOQOL Group. The tool was tried by mastering students of Production Engineering and associate students of Analysis and Development of Systems of UTFPR. It also was submitted to a simulation with the SPSS, where the obtained results were exactly the same. The syntax used in the tool construction is available, enabling its adaptation for application in other instruments of evaluation. It concludes that, from the present paper, was possible to make
\end{abstract}


available a free tool, developed in a widely used platform and that allows applications of WHOQOL-100 without needing the SPSS's utilization.

Key-words: Quality of Life, Health Evaluation; World Health Organization, WHOQOL-100.

\section{Introdução}

O termo saúde foi, em 1946, definido pela Organização Mundial da Saúde (OMS) como "um completo estado de bem-estar físico, mental e social e não meramente a ausência de doença" (FLECK et al. 2000). Ainda que date de mais de meio século a quebra do paradigma de que a saúde é o oposto de enfermo, a preocupação com a melhoria da saúde se intensificou em meados da década de 70.

Mesmo havendo conformidade de opiniões sobre a importância de se avaliar a qualidade de vida, tal conceito apresenta dissensão. A multidimensionalidade do conceito de qualidade de vida abarca diferentes indicadores, propostos por autores que conformaram diferentes construções conceituais. Tal fato faz com que o peso atribuído aos indicadores promova a existência de conceitos distintos (FLECK, 2008).

$O$ fato de não haver um consenso conceitual constitui um grande problema no desenvolvimento de instrumentos de avaliação da qualidade de vida, ao passo que não é possível afirmar com clareza quais elementos estes instrumentos estão mensurando (FLECK, 2008).

Partindo dessa premissa, o ponto de partida para a construção do instrumento de avaliação da qualidade de vida da OMS, foi conceituar qualidade de vida. No conceito construído, qualidade de vida foi entendida como "a percepção do indivíduo de sua posição na vida no contexto da cultura e sistema de valores nos quais ele vive e em relação aos seus objetivos, expectativas, padrões e preocupações" (FLECK et al., 1999a, p. 199). Pautado nesse conceito, a OMS empenhou-se na construção dos instrumentos WHOQOL, que avaliam a qualidade de vida tanto de forma global (WHOQOL-100 e WHOQOL-bref) como também em função de grupos específicos (WHOQOL120-HIV, WHOQOL-HIV-bref, WHOQOL-OLD, WHOQOL-SRPB).

O WHOQOL-100 é o instrumento de avaliação da qualidade de vida mais utilizado no mundo. Atualmente os instrumentos WHOQOL estão disponíveis em mais de 50 idiomas (WHO Field Centre for the Study of Quality of Life of Bath, 2008).

Para a obtenção dos resultados das aplicações dos instrumentos WHOQOL, o Grupo WHOQOL recomenda o software Statistical Package for the Social Sciences (SPSS), um software estatístico que exige conhecimentos específicos para a sua utilização. O SPSS não é de distribuição gratuita.

Nessa perspectiva, o presente estudo tem como objetivo - seguindo a sintaxe proposta pelo Grupo WHOQOL - a elaboração de uma ferramenta para realizar o cálculo dos escores e a estatística descritiva do WHOQOL-100 a partir do software Microsoft Excel, um software de ampla acessibilidade. Como objetivo específico, tem-se a proposta de clarificar os procedimentos para o cálculo dos escores do WHOQOL-100, os quais estão dispostos implicitamente nos documentos publicados pelo Grupo WHOQOL. A consecução dos objetivos propostos possibilita uma ascensão da utilização de um instrumento diretamente relacionado com a área objeto de estudo desse trabalho, a Saúde Pública.

\section{O instrumento WHOQOL-100}

\subsection{Desenvolvimento do WHOQOL-100}

O desenvolvimento do Instrumento de Avaliação da Qualidade de Vida da OMS foi realizado em 15 centros simultaneamente, sediados em 14 países. Após o desenvolvimento do projeto WHOQOL, novos centros foram incorporados e o WHOQOL-100 foi disponibilizado em mais de 20 idiomas. 
A metodologia de desenvolvimento do WHOQOL foi seccionada em quatro grandes etapas: clarificação do conceito de qualidade de vida, estudo piloto qualitativo, desenvolvimento de um piloto e uma aplicação de campo. Para os centros integrados, após a conclusão da elaboração do instrumento, foi estabelecida uma metodologia que consistia na sua tradução, preparação do teste piloto, desenvolvimento das escalas de respostas e administração do piloto (Grupo WHOQOL, 1998).

A versão em português do WHOQOL-100 foi desenvolvida pelo Departamento de Psiquiatria e Medicina Legal da Faculdade de Medicina (FAMED), da Universidade Federal do Rio Grande do Sul (UFRGS), sob a coordenação do professor Marcelo Pio de Almeida Fleck. Apesar de não estar incluída dentre os 15 centros iniciais do desenvolvimento do WHOQOL, o centro de Porto Alegre-RS foi um dos primeiros centros a se incorporar ao Grupo WHOQOL (Grupo WHOQOL, 1998).

\subsection{Elaboração das questões do WHOQOL-100}

A partir das sugestões formuladas por cada um dos centros de desenvolvimento, totalizou-se aproximadamente 1800 questões. Após a eliminação das questões redundantes, equivalentes ou que não se aplicavam ao instrumento, o número de questões foi reduzido para 1000. Fora realizado, então, uma classificação das perguntas com relação à qualidade de vida e cultura, em cada centro de desenvolvimento (FLECK et al., 1999b).

Ao combinar todas as questões, foram selecionadas 235 questões, seccionadas em 29 facetas, para o instrumento piloto do WHOQOL-100. Segundo Fleck et al. (1999b, p. 24), os critérios para a elaboração do WHOQOL-100 foram:

- Basear-se tanto quanto possível nas sugestões dos pacientes e profissionais de saúde participantes dos grupos focais;

- Proporcionar respostas que esclareçam acerca da qualidade de vida dos respondentes, como definida pelo projeto;

- Refletir o significado proposto pela definição das facetas;

- Cobrir em combinação com outras questões para uma dada faceta, os aspectos chaves de cada faceta como descritas na definição destas;

- Usar linguagem simples, evitando ambigüidade nas palavras e frases;

- Preferir questões curtas em relação às longas;

- Evitar duas negações;

- Serem compatíveis com uma escala de avaliação;

- Explorar um só problema por faceta;

- Evitar as referências explícitas em relação a tempo ou outro termo de comparação (p.ex. o ideal, ou antes de eu estar doente);

- Ser aplicável a indivíduos com vários graus de disfunção;

- Ser formuladas como questões e não como afirmações;

- Refletir a tipologia das questões adotadas no projeto.

Após a aplicação do teste piloto, foram selecionadas as melhores questões para cada faceta, de forma a estabelecer a consistência interna e validade discriminante do instrumento. Foram selecionadas 100 questões seccionadas em 24 facetas. As facetas foram agrupadas em seis grandes domínios (FLECK et al., 1999b).

\subsection{Escalas de respostas do WHOQOL-100}

Todas as questões do WHOQOL-100 são fechadas. É utilizada uma escala de respostas do tipo Likert, composta por cinco elementos, variando entre 1 e 5. Esses extremos representam $0 \%$ e $100 \%$, respectivamente. Existem quatro tipos diferentes de escala de respostas, conforme pode ser observado no Quadro 1: 
Quadro 1 - Escalas de respostas do WHOQOL-100

\begin{tabular}{|c|c|c|c|c|c|}
\hline ESCALA & $\mathbf{0 \%}$ & $\mathbf{2 5 \%}$ & $\mathbf{5 0 \%}$ & $\mathbf{7 5 \%}$ & $\mathbf{1 0 0 \%}$ \\
\hline INTENSIDADE & nada & muito pouco & mais ou menos & bastante & extremamente \\
\hline \multirow{3}{*}{ AVALIAÇÃO } & $\begin{array}{c}\text { muito } \\
\text { insatisfeito }\end{array}$ & insatisfeito & $\begin{array}{c}\text { nem satisfeito } \\
\text { nem insatisfeito }\end{array}$ & satisfeito & $\begin{array}{c}\text { muito } \\
\text { satisfeito }\end{array}$ \\
\cline { 2 - 6 } & muito ruim & ruim & $\begin{array}{c}\text { nem ruim } \\
\text { nem bom }\end{array}$ & bom & muito bom \\
\cline { 2 - 6 } & muito infeliz & infeliz & $\begin{array}{c}\text { nem feliz } \\
\text { nem infeliz }\end{array}$ & feliz & muito feliz \\
\hline CAPACIDADE & nada & muito pouco & médio & muito & completamente \\
\hline FREQÜÊNCIA & nunca & raramente & às vezes & repetidamente & sempre \\
\hline
\end{tabular}

Fonte: Adaptado de Grupo WHOQOL (1998)

A distribuição das escalas de respostas do WHOQOL-100 pode ser verificada na Figura 1:

Figura 1 - Distribuição das escalas de respostas do WHOQOL-100

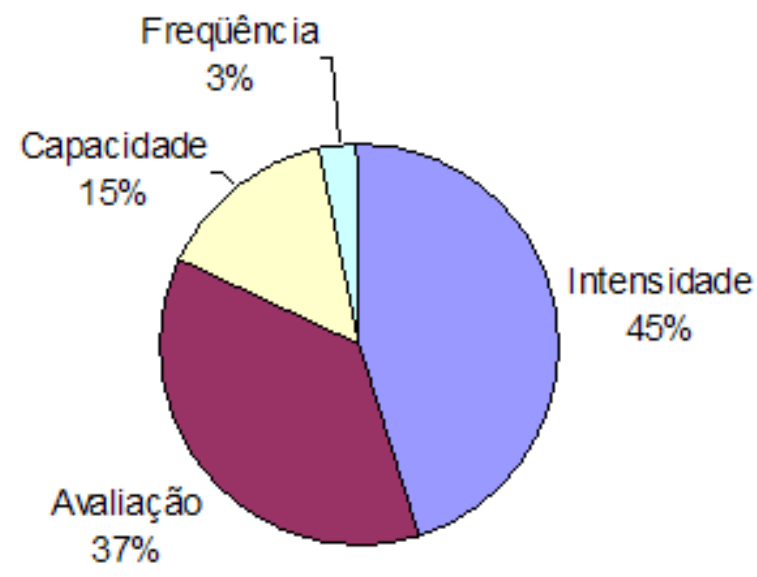

Fonte: Autoria própria

É perceptível o predomínio das respostas com escala de intensidade e avaliação, enquanto as respostas com escala de capacidade e freqüência estão presentes em menor número. Dentre as três formas de resposta previstas na escala de avaliação, há predominância das questões relacionadas à satisfação (muito insatisfeito - muito satisfeito) com 29 incidências, seguido pelas questões com enfoque absoluto na avaliação (muito ruim - muito bom) com sete incidências, e finalizando com uma única resposta que faz menção à felicidade (muito infeliz - muito feliz).

\subsection{Cálculo do escore do WHOQOL-100}

Os resultados da aplicação do WHOQOL-100 são expressos através dos escores de cada faceta e domínio. É realizado, também, o cálculo da estatística descritiva de cada faceta e domínio. A OMS aconselha a utilização do software estatístico SPSS para o cálculo dos resultados do WHOQOL-100.

Mesmo que a sintaxe SPSS para o WHOQOL-100 seja de domínio público, o Grupo WHOQOL apenas divulga as linhas de código da sintaxe, sem a preocupação de explicar os critérios para o cálculo dos escores dos domínios e facetas do WHOQOL-100. Na tentativa de suprimir essa omissão, neste estudo foram traduzidas as linhas de código da sintaxe SPSS, explicando quais são os procedimentos utilizados para o cálculo dos escores dos domínios e facetas do WHOQOL-100. Assim, pode-se afirmar que os resultados do WHOQOL-100 são calculados da seguinte forma: 
- É verificado se todas as 100 questões foram preenchidas com valores entre 1 e 5;

- Invertem-se as 18 questões cuja escala de respostas é invertida;

- Os escores das facetas são calculados a partir da média aritmética simples das questões que compõem cada faceta, seguido de uma multiplicação por quatro. A multiplicação por quatro é utilizada para que, no caso de uma questão não ter sido respondida, o escore da faceta compense a nulidade da questão através do produto pelo número de questões válidas que a faceta deveria ter. Serão computadas somente as facetas que possuírem pelo menos três itens válidos;

- São calculados os escores dos domínios através da média aritmética simples entre os escores das facetas que compõem cada domínio. Nos domínios compostos por até cinco facetas, este será calculado somente se o número de facetas não calculadas não for igual ou superior a dois. Nos domínios compostos por mais de cinco facetas, este será calculado somente se o número de facetas não calculadas não for igual ou superior a três. No caso de facetas em escala invertida (todas as questões pertencentes à faceta possuem escala de respostas invertida), realiza-se a inversão dessa faceta para o prosseguimento do cálculo;

- Realiza-se uma contagem do total de itens respondidos por cada respondente. São computados no cálculo somente os respondentes que preencheram corretamente pelo menos 80 itens ( $80 \%$ dos itens do instrumento);

- Por fim, é realizada a estatística descritiva de cada faceta e domínio. Os elementos calculados são: média, desvio padrão, valor mínimo e valor máximo.

Os resultados do WHOQOL-100 são expressos em uma escala variante entre 4 e 20 pontos. Tal escala se deve ao fato do cálculo do escore das facetas ser realizado através da multiplicação da média das questões que constituem cada faceta por quatro. Como cada domínio é calculado através da média aritmética simples das facetas que o compõem, os resultados são expressos na mesma escala das facetas.

Embora não conste na sintaxe proposta pela equipe responsável pela tradução e validação do WHOQOL no Brasil, o Manual do Usuário do WHOQOL (The WHOQOL Group, 1998) propõe a conversão dos resultados para uma escala de 0 a 100.

\subsection{Conversão da escala de respostas das questões e facetas invertidas}

A conversão das questões é utilizada com o objetivo de padronizar todas as respostas do instrumento, de forma que a resposta mais positiva seja 5. Por conseguinte, a resposta mais negativa deve ser 1. Desta forma, todas as questões de cada faceta são convertidas para uma mesma escala, onde o aumento gradativo da resposta equivale, na mesma proporção, ao aumento no positivismo do resultado da faceta.

No caso de todas as quatro questões que constituem uma faceta sejam dispostas em escala invertida, essa mesma lógica é utilizada, mas somente no cálculo do domínio. Ou seja, o resultado dessas facetas é expresso na escala original, sem inversão. Entretanto, ao se calcular o escore dos domínios nos quais tais facetas estão inseridas, o escore dessas é convertido.

Para a conversão da escala de resposta das questões, o valor mínimo da questão de escala invertida deve ser substituído pelo valor máximo da questão de escala normal, assim como o valor máximo da questão de escala invertida deve ser substituído pelo valor mínimo da questão de escala normal. O mesmo deve ocorrer com os valores intermediários, seguindo essa mesma lógica. Assim, o único valor que não é alterado é o valor central, que tanto na escala normal, quanto na escala invertida, permanece o mesmo.

É preciso estar atento a esse fato, pois ao comparar os resultados entre as facetas, o escore de uma faceta de escala invertida não pode ser diretamente comparado ao escore de uma faceta de escala normal. Pode se observar no Quadro 2 os valores assumidos pelas respostas de questões cuja escala é invertida: 
Quadro 2 - Conversão de escala

\begin{tabular}{|l|c|c|c|c|c|}
\hline TIPO DE ESCALA & $\mathbf{0 \%}$ & $\mathbf{2 5 \%}$ & $\mathbf{5 0 \%}$ & $\mathbf{7 5 \%}$ & $\mathbf{1 0 0 \%}$ \\
\hline Normal & 1 & 2 & 3 & 4 & 5 \\
\hline Invertida & 5 & 4 & 3 & 2 & 1 \\
\hline \multicolumn{7}{|c|}{ Fonte: autoria própria }
\end{tabular}

O mesmo procedimento é utilizado na conversão de facetas invertidas. Após a conversão de uma faceta negativa, os valores "âncora" 4, 8, 12, 16 e 20 passam a assumir os valores 20, 16, 12, 8 e 4, respectivamente. Valores intermediários são convertidos na mesma proporção.

\subsection{Questões, domínios e facetas do WHOQOL-100}

O WHOQOL-100 é seccionado em 24 grupos de quatro questões cada, recebendo a denominação de "facetas". Por sua vez, o conjunto de facetas constitui um "domínio". Ao contrário da composição das facetas, os seis domínios do WHOQOL-100 não são constituídos pelo mesmo número de facetas, podendo variar de um até oito facetas.

As questões que compõem o WHOQOL-100 não estão dispostas no questionário seguindo uma seqüência lógica por domínio ou por faceta. A distribuição das facetas e domínios do WHOQOL-100 estão relacionadas no Quadro 3:

Quadro 3-Domínios e facetas dos WHOQOL-100

\begin{tabular}{|c|c|}
\hline DOMÍNIOS & FACETAS \\
\hline \multirow{3}{*}{ Domínio I - Domínio físico } & 1. Dor e desconforto \\
\hline & 2. Energia e fadiga \\
\hline & 3. Sono e repouso \\
\hline \multirow{5}{*}{ Domínio II - Domínio psicológico } & 4. Sentimentos positivos \\
\hline & 5. Pensar, aprender, memória e concentração \\
\hline & 6. Auto-estima \\
\hline & 7. Imagem corporal e aparência \\
\hline & 8. Sentimentos negativos \\
\hline \multirow{4}{*}{ Domínio III - Nível de Independência } & 9. Mobilidade \\
\hline & 10. Atividades da vida cotidiana \\
\hline & 11. Dependência de medicação ou de tratamentos \\
\hline & 12. Capacidade de trabalho \\
\hline \multirow{3}{*}{ Domínio IV - Relações sociais } & 13. Relações pessoais \\
\hline & 14. Suporte (Apoio) social \\
\hline & 15. Atividade sexual \\
\hline \multirow{8}{*}{ Domínio V-Ambiente } & 16. Segurança física e proteção \\
\hline & 17. Ambiente no lar \\
\hline & 18. Recursos financeiros \\
\hline & 19. Cuidados de saúde e sociais: disponibilidade e qualidade \\
\hline & 20. Oportunidades de adquirir novas informações e habilidades \\
\hline & 21. Participação em, e oportunidades de recreação/lazer \\
\hline & 22. Ambiente físico: (poluição/ruído/trânsito/clima) \\
\hline & 23. Transporte \\
\hline $\begin{array}{l}\text { Domínio VI- Aspectos } \\
\text { espirituais/Religião/Crenças pessoais }\end{array}$ & 24. Espiritualidade/religião/crenças pessoais \\
\hline
\end{tabular}

Fonte: Grupo WHOQOL (1998)

O WHOQOL-100 possui uma faceta que não está inserida em nenhum domínio, a faceta "Qualidade de vida global e percepção geral da saúde" (The WHOQOL Group, 1998). Esta faceta aborda uma auto-avaliação da qualidade de vida, onde o respondente avalia a satisfação com a sua vida, saúde e qualidade de vida. 


\section{Procedimentos da construção da ferramenta}

Na perspectiva de tornar a ferramenta proposta passível de reprodução e possibilitando que a mesma lógica seja adaptada para outros instrumentos, a sintaxe utilizada na confecção é disponibilizada. Cada linha de código é seguida por uma breve explicação sobre a lógica empregada na mesma.

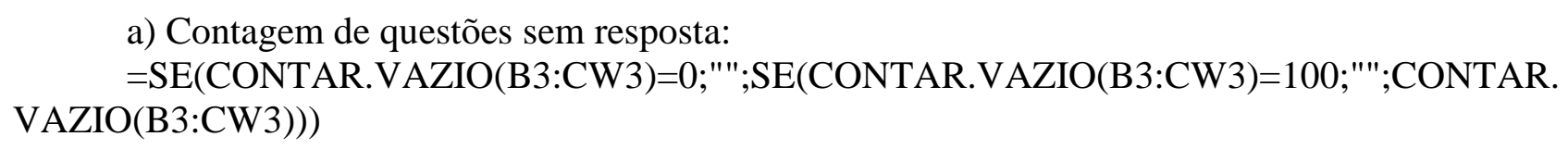

a) Contagem de questões sem resposta:

=SE(CONTAR.VAZIO(B3:CW3)=0;"";SE(CONTAR.VAZIO(B3:CW3)=100;"';CONTAR. $\mathrm{VAZIO}(\mathrm{B} 3: \mathrm{CW} 3)))$

em que B3 e CW3 representam, respectivamente, a primeira e a centésima questão do instrumento. Caso o número de questões sem resposta seja nulo ou igual a 100, a célula não retorna nenhum resultado. Se houver alguma questão sem resposta o número de questões não respondidas será expresso.

b) Contagem de questões respondidas incorretamente:

$=$ SE $(C O N T A R . V A Z I O(B 3: C W 3)=100 ; "$; SE $(($ CONT.SE $(B 3: C W 3 ; ">5 "))+(C O N T . S E(B 3:$ BW3;"<1")+(CONT.SE(B3:CW3;"*"))) $=0 ;$;";(CONT.SE(B3:CW3;">5"))+(CONT.SE(B3:BW3;" $<1 "))+(\mathrm{CONT} . \mathrm{SE}(\mathrm{B} 3: \mathrm{CW} 3 ; " * "))))$

onde é verificado se alguma questão foi respondida com um valor numérico não situado no intervalo compreendido entre 1 e 5 , ou respondida com um valor não numérico. O número de questões não respondidas sempre deve ser nulo. Caso alguma irregularidade seja constatada, o pesquisador deve obrigatoriamente corrigi-la.

c) Critérios de eliminação de respondentes:

$=$ SE $($ E $(C O N T A R . V A Z I O(B 3: C W 3)>20 ; C O N T A R . V A Z I O(B 3: C W 3)<=99) ; " R E M O V E R$ RESPONDENTE";SE(CONTAR.VAZIO(B3:CW3)=100;"';SE(EI3="";"REMOVER_RESPONDE NTE";"")))

onde o pesquisador é instruído a excluir o respondente caso o número de questões não respondidas seja superior a 20, ou se dois ou mais domínios não puderam ser calculados. Caso seja constatado que o respondente deve, efetivamente, ser removido da amostra, a célula retorna a mensagem "remover respondente".

d) Cálculo dos escores das facetas:

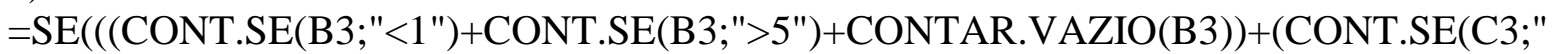
$<1 ")+$ CONT.SE(C3;">5")+CONTAR.VAZIO(C3))+(CONT.SE(D3;"<1")+CONT.SE(D3;">5")+C ONTAR.VAZIO(D3))+(CONT.SE(CI3;"<1")+CONT.SE(CI3;">5")+CONTAR.VAZIO(CI3)))>=2 ;"';(MÉDIA(B3;C3;D3;CI3))*4)

em que B3, C3, D3 e CI3 representam as quatro questões pertencentes à faceta 1 . A faceta é calculada somente se pelo menos três dessas quatro questões tiverem sido respondidas corretamente.

e) Cálculo dos escores das facetas compostas por questões com escala de respostas invertida: $=\mathrm{SE}(((\mathrm{CONT} . \mathrm{SE}(\mathrm{E} 3 ; "<1 ")+\mathrm{CONT} . \mathrm{SE}(\mathrm{E} 3 ; ">5 ")+\mathrm{CONTAR} . \mathrm{VAZIO}(\mathrm{E} 3))+(\mathrm{CONT} . \mathrm{SE}(\mathrm{F} 3 ; "$ $<1 ")+$ CONT.SE(F3;">5")+CONTAR.VAZIO(F3))+(CONT.SE(AO3;"<1")+CONT.SE(AO3;">5") +CONTAR.VAZIO(AO3))+(CONT.SE(BE3;"<1")+CONT.SE(BE3;">5")+CONTAR.VAZIO(BE3 )) )>=2;"";(MÉDIA(SE(E3="";MÉDIA(6-F3;AO3;BE3);6-E3);SE(F3="";MÉDIA(6$\left.\mathrm{E} 3 ; \mathrm{AO} 3 ; \mathrm{BE} 3) ; 6-\mathrm{F} 3) ; \mathrm{AO} 3 ; \mathrm{BE} 3))^{*} 4\right)$

em que E3, F3, AO3 e BE3 representam as quatro questões pertencentes à faceta 2. A faceta é calculada somente se no mínimo três dessas quatro questões tiverem sido respondidas corretamente. As questões cuja escala de respostas é invertida devem ser subtraídas de seis unidades. Seguindo a lógica exemplificada na faceta 1 , caso alguma questão invertida não seja respondida, esta recebe o valor 6 (seis menos zero é igual a seis). Para contornar esse erro, deve ser especificado que, se uma questão invertida não for respondida, essa questão deve ser excluída do cálculo da faceta.

f) Cálculo dos escores dos domínios: 


\section{(DN3:DP3)))}

$=$ SE(CONTAR.VAZIO(DN3:DP3)>=2;"';SE(CONTAR.VAZIO(B3:CW3)>=20;"';MÉDIA

em que DN3 e DP3 representam, respectivamente, a primeira e a última faceta pertencente ao domínio 4. O domínio será calculado somente se o número de facetas não calculadas for inferior a dois (exceto no domínio 5, onde este é calculado somente se o número de facetas não calculadas for inferior a três).

g) Cálculo dos escores dos domínios compostos por facetas invertidas:

=SE(CONTAR.VAZIO(DB3:DD3)>=2;"";SE(CONTAR.VAZIO(B3:CW3)>=20;"';MÉDI A(SE(DB3="";MÉDIA(DC3;DD3);24-DB3);DC3;DD3)))

em que DB3, DC e DD3 representam as facetas pertencentes ao domínio 1. O domínio será calculado somente se o número de facetas não calculadas for inferior a dois (exceto no domínio 5 , onde este é calculado somente se o número de facetas não calculadas for inferior a três). As facetas invertidas devem ser subtraídas de 24 unidades, em virtude do mesmo problema notificado no cálculo dos escores das facetas compostas por questões com escala de respostas invertida.

h) Cálculo do escore "Total" da Qualidade de Vida:

=SE(CONTAR.VAZIO(EB3:EG3)>=2;"';SE(E(DB3="";DI3="";DL3="");MÉDIA(DC3:D

H3;DJ3;DK3;DM3:DZ3);SE(E(DB3="";DI3="");MÉDIA(DC3:DH3;DJ3;24-

DL3;DK3;DM3:DZ3);SE(E(DI3="";DL3="");MÉDIA(24-

DB3;DC3:DH3;DJ3;DK3;DM3:DZ3);SE(E(DB3="";DL3="");MÉDIA(DC3:DH3;DJ3;24-

DI3;DK3;DM3:DZ3);MÉDIA(SE(DB3=""';MÉDIA(DC3:DH3;24-DI3;DJ3;DK3;24-

DL3;DM3:DZ3);24-DB3);SE(DI3="";MÉDIA(24-DB3;DC3:DH3;DJ3;DK3;24-

DL3;DM3:DZ3);24-DI3);SE(DL3=""'MÉDIA(24-DB3;DC3:DH3;24-DI3;DJ3;DK3;DZ3);24-

DL3);DC3:DH3;DJ3;DK3;DM3:DZ3))))))

em que EB3 e EG3 representam, respectivamente o domínio 1 e o domínio 6 e DB, DC, DD, DE, DF, DG, DH, DI, DJ, DK, DL, DM, DN, DO, DP, DQ, DR, DS, DT, DU, DV, DW, DX, DY e DZ representam as 25 facetas. O escore total é calculado somente se o número de domínios não calculados for menor do que dois. As facetas invertidas devem ser subtraídas de 24 unidades.

i) Cálculo da estatística descritiva de uma variável (média, desvio padrão, coeficiente de variação, valor mínimo, valor máximo e amplitude):

=MÉDIA(Tabulação!B3:B1002)

=DESVPAD $($ Tabulação!B3:B1002)

$=(($ DESVPAD $($ Tabulação!B3:B1002) $) /($ MÉDIA $($ Tabulação!B3:B1002) $)) * 100$

=MÍNIMO(Tabulação!B3:B1002)

=MÁXIMO(Tabulação!B3:B1002)

=(MÁXIMO(Tabulação!B3:B1002) $)-($ MÍNIMO(Tabulação!B3:B1002) $)$

em que B3 e B1002 representam, respectivamente, a resposta/escore de determinada questão, faceta, domínio ou total do primeiro e do último respondente.

A ferramenta foi construída a partir do software Microsoft Excel 2003, sendo também testada nas versões 2000, XP e 2007. Os resultados obtidos foram os mesmos em todas as versões.

Para garantir a adequação da ferramenta ao seu propósito, esta fora encaminhada para dois pesquisadores do Mestrado em Engenharia de Produção da UTFPR testarem a sua utilização. Foi encaminhada, também, para dois alunos de graduação do Curso Superior de Tecnologia em Análise e Desenvolvimento de Sistemas da UTFPR verificarem a existência de erros em sua sintaxe. As sugestões provenientes dos pesquisadores foram avaliadas e, quando pertinentes, acatadas. Os erros constatados foram devidamente corrigidos.

\section{Resultados}

A ferramenta proposta realiza automaticamente todos os cálculos propostos pelo Grupo WHOQOL, sendo que o pesquisador que utilizá-la precisa apenas preencher as respostas concedidas pelos respondentes nas células especificadas. Para o cálculo do escore das facetas, utiliza-se dos mesmos critérios de exclusão de respondentes propostos pelo WHOQOL, com 
algumas modificações que serão apresentadas na seqüência, objetivando facilitar o preenchimento e detecção de erros. A lógica utilizada pela ferramenta é a seguinte:

- As células cujas respostas estão corretas (respondidas com valores situados no intervalo compreendido entre 1 e 5) são representadas através da cor de preenchimento verde;

- Caso algum respondente tenha deixado de responder 20 ou mais questões, o pesquisador será instruído para excluir tal respondente. O número de questões não respondidas é informado ao pesquisador e as questões não respondidas estarão em destaque (cor do preenchimento branco);

- Caso alguma resposta tenha sido preenchida com algum valor que não conste entre o intervalo de 1 a 5, o número de respostas inválidas será notificado ao pesquisador e as respostas inválidas estarão em destaque (cor do preenchimento vermelho);

- As questões de escala invertida são devidamente convertidas;

- Se somente três das quatro questões que compõem uma faceta tenham sido respondidas corretamente, a faceta será calculada através da média das respostas dessas questões seguido de uma multiplicação por quatro;

- Se duas ou mais questões de uma mesma faceta não forem respondidas ou tenham sido respondidas com um valor que não conste no intervalo de 1 a 5 , o escore de tal faceta não é calculado;

- Em domínios compostos por até cinco facetas, se duas ou mais facetas pertencentes ao mesmo domínio não tiverem sido pontuadas (em virtude do item anterior), o escore desse domínio não será calculado. Em domínios compostos por mais de cinco facetas, se três ou mais facetas pertencentes a este domínio não tiverem sido pontuadas, o escore desse domínio não será calculado;

- Caso dois ou mais domínios não sejam calculados, o pesquisador é instruído a excluir o respondente da amostra;

- É calculado um escore "Total" do respondente. Proposto neste trabalho, tal escore consiste no cálculo da média aritmética simples dos escores das 25 facetas;

- A estatística descritiva de cada questão, faceta, domínio e "Total" são calculados. Os valores apresentados na estatística descritiva são: média, desvio padrão, valor máximo, valor mínimo, coeficiente de variação e amplitude (os dois últimos foram adicionados pelo autor);

- As médias dos escores das facetas e domínios são convertidas em uma escala de 0 a 100 , e são exibidas em um gráfico.

Após a inserção dos dados, para a utilização dos resultados de sua pesquisa, o pesquisador poderá copiar os escores individuais de cada respondente, os resultados da estatística descritiva e os gráficos, entretanto, não pode modificar tais resultados. A única área que lhe é permitida a inserção e edição de valores é a área de tabulação das respostas dos respondentes.

Para a validação da ferramenta, foram realizadas simulações com dados reais de aplicações do WHOQOL-100, comparando os resultados obtidos através da ferramenta proposta com os obtidos no SPSS. Os resultados retornados por ambos os softwares foram exatamente os mesmos, garantindo assim a fidedignidade da ferramenta objeto de estudo deste trabalho.

Os requisitos para a utilização da presente ferramenta são: computador ou notebook com o software Microsoft Excel instalado. O download da ferramenta pode ser realizado através da URL: http://www.brunopedroso.com.br/bpwhoqol-100.xls.

\section{Considerações finais}

O WHOQOL-100 é o instrumento de avaliação da qualidade de vida mais utilizado no mundo. Mesmo sendo um instrumento amplamente difundido, a dificuldade de interpretação de sua sintaxe e o emprego do software SPSS para o cálculo dos resultados, são fatores limitantes para sua utilização. 
Almejando a supressão dessas limitações, o presente estudo transcreveu textualmente a sintaxe SPSS, detalhando todos os passos utilizados para a obtenção dos resultados do WHOQOL100. Foi, também, construída uma ferramenta, a partir do software Microsoft Excel 2003, para a realização do cálculo dos escores e estatística descritiva do WHOQOL-100.

A ferramenta foi testada em diferentes versões desse software - 2000, XP e 2007. Constatou-se a sua compatibilidade com todas as versões testadas, sem divergências nos resultados. Outro procedimento adotado foi o teste por pesquisadores do Mestrado em Engenharia de Produção e do Curso Superior de Tecnologia em Análise e Desenvolvimento de Sistemas da UTFPR. Os resultados obtidos foram idênticos aos da sintaxe proposta pela OMS para o software SPSS.

Na tentativa de tornar a ferramenta passível de reprodução, e, perspectivando a possibilidade da lógica empregada na construção da ferramenta ser aplicada em outros instrumentos, foi disponibilizado partes da sintaxe desenvolvida, o que possibilita seu desenvolvimento completo. A sintaxe é seguida por uma clarificação das variáveis e da lógica utilizada em cada linha de código.

\section{Referências}

FLECK, M. P. A. O instrumento de avaliação de qualidade de vida da Organização Mundial da Saúde (WHOQOL-100): características e perspectivas. Ciência e Saúde Coletiva. v. 5, n. 1, p.33$38,2000$.

FLECK, M. P. A. et al. Aplicação da versão em português do instrumento de avaliação de qualidade de vida da Organização Mundial da Saúde (WHOQOL-100). Revista de Saúde Pública. v. 33, n. 2, p. 198-205, 1999a.

FLECK, M. P. A. et al. Desenvolvimento da versão em português do instrumento de avaliação de qualidade de vida da OMS (WHOQOL-100). Revista Brasileira de Psiquiatria. v. 21, n. 1, p.1928, 1999 b.

FLECK, M. P. A. et al. Problemas conceituais em qualidade de vida. In: FLECK, M. P. A. et al. A avaliação de qualidade de vida: guia para profissionais da saúde. Porto Alegre: Artmed, 2008.

Grupo WHOQOL. Versão em português dos instrumentos de avaliação da qualidade de vida (WHOQOL) 1998. Disponível em <http://www.ufrgs.br/psiq/whoqol1.html>. Acesso em: 13 abr. 2008.

WHO Field Centre for the Study of Quality of Life of Bath. About the WHO Field Centre for the Study of Quality of Life. Disponível em: <http://www.bath.ac.uk/whoqol/about.cfm>. Acesso em: 13 abr. 2008.

The WHOQOL Group. WHOQOL User Manual. Geneva: 1998. 